\title{
Vascular risk factors, atherosclerosis, cerebral white matter lesions and cerebral perfusion in a population-based study
}

\author{
J.J. Claus ${ }^{1}{ }^{*}$, M.M.B. Breteler1, D. Hasan², E.P. Krenning ${ }^{3}$, M.L. Bots ${ }^{1}$, D.E. Grobbee ${ }^{1}$, J.C. van Swieten², \\ F. van Harskamp², A. Hofman ${ }^{1}$ \\ 1 Department of Epidemiology and Biostatistics, Erasmus University Medical School, Rotterdam, The Netherlands \\ 2 Department of Neurology, University Hospital Rotterdam Dijkzigt, Rotterdam, The Netherlands \\ ${ }^{3}$ Department of Nuclear Medicine, University Hospital Rotterdam Dijkzigt, Rotterdam, The Netherlands
}

Received 1 January and in revised form 20 February 1996

\begin{abstract}
We studied risk factors for cerebral vascular disease (blood pressure and hypertension, factor VIIc, factor VIIIc, fibrinogen), indicators of atherosclerosis (intima-media thickness and plaques in the carotid artery) and cerebral white matter lesions in relation to regional cerebral blood flow (rCBF) in 60 persons (aged 65-85 years) recruited from a population-based study. $\mathrm{rCBF}$ was assessed with single-photon emission tomography using technetium- $99 \mathrm{~m} \quad d, l$-hexamethylpropylene amine oxime ( $\left.{ }^{99 m} \mathrm{Tc}-\mathrm{HMPAO}\right)$. Statistical analysis was performed with multiple linear regression with adjustment for age, sex and ventricle-to-brain ratio. A significant positive association was found between systolic and diastolic blood pressure and temporo-parietal rCBF. In analysis with quartiles of the distribution, we found a threshold effect for the relation of low diastolic blood pressure $(\leq 60 \mathrm{mmHg})$ and low temporo-parietal rCBF. Levels of plasma fibrinogen were inversely related to parietal $\mathrm{rCBF}$, with a threshold effect of high fibrinogen levels $(>3.2 \mathrm{~g} / \mathrm{l})$ and low rCBF. Increased atherosclerosis was related to low $\mathrm{rCBF}$ in all cortical regions, but these associations were not significant. No consistent relation was observed between severity of cerebral white matter lesions and $\mathrm{rCBF}$. Our results may have implications for blood pressure control in the elderly population.
\end{abstract}

Key words: Vascular risk factors - Atherosclerosis - Cerebral white matter lesions - Cerebral blood flow - Elderly population

Eur J Nucl Med (1996) 23:675-682

\footnotetext{
* Present address: Department of Neurology, Academic Medical Center, Meibergdreef 9, 1105 AZ Amsterdam, The Netherlands
}

Correspondence to: J.J. Claus

\section{Introduction}

Several studies have related the presence of vascular risk factors to changes in regional cerebral blood flow ( $\mathrm{CCBF})$. An overall CBF decrease was reported in persons with vascular risk factors compared to age-matched controls without these risk factors [1] and some authors have suggested an association of vascular risk factors with CBF, including hypertension [2], whole blood viscosity [3] and smoking [4]. Clinical indicators of atherosclerosis have also been linked to decreases in rCBF [5, 6]. However, few reports are available on the relation between indicators of vascular risk and atherosclerosis and $\mathrm{rCBF}$ in the general population.

The reported frequency of cerebral white matter lesions in the elderly on magnetic resonance imaging (MRI) scans varies around 25\% [7-9] and these lesions may be associated with cognitive impairment of subcorticofrontal functions in a random sample of non-demented elderly subjects [10]. Reductions in CBF investigated with positron emission tomography (PET) and the xenon-133 inhalation technique have been reported in elderly individuals with severe white matter abnormalities $[11,12]$. Others, however, did not find a significant relation between periventricular white matter lesions on MRI and CBF measured with the ${ }^{133} \mathrm{Xe}$ inhalation technique [13-15]. Since subjects included in some of these studies were selected on the basis of presence or absence of cardiovascular risk factors, these inconsistent results leave open the question of whether, in the general population, lesions in cerebral white matter are associated with decrements of $\mathrm{rCBF}$ in cerebral grey matter.

In this study, we investigated in a population-based sample the association of $\mathrm{rCBF}$, measured with singlephoton emission tomography (SPET) using technetium$99 \mathrm{~m} \quad d, l$-hexamethylpropylene amine oxime (99mTcHMPAO), and vascular risk factors, including thrombogenic factors, indicators of atherosclerosis and cerebral white matter lesions on MRI. 


\section{Materials and methods}

Subjects. This study is based on a population-based study (The Rotterdam Study) [16] for which all persons of 55 years and over from the suburb of Ommoord in Rotterdam were eligible, including institutionalized persons. The response rate of the main study was $79 \%$ and written informed consent was obtained for participation in this study as well as the SPET investigation. Subjects for the present study were recruited from a group of 111 persons randomly selected as a stratified sample of persons from 65 to 85 years old who participated in an MRI study (response rate 87\%) [9]. In this MRI study three persons were excluded because they had a pacemaker or metal prostheses or clips, one because he was suffering from a major psychiatric disorder and two because they were wheelchair-bound. An additional 21 persons were excluded from the SPET study because they had a neurological disorder with a known effect on rCBF, including a history of transient ischaemic attacks (TIAs) or stroke, Parkinson's disease or dementia, including Alzheimer's disease (employing a careful screening for dementia [17] and NINCDS-ADRDA criteria) [18]. Twenty-four of the 90 eligible persons refused participation and one died before the SPET study could be performed. Five scans were not available for technical reasons and a total of 60 subjects were included in the present study.

Measurements. At a home interview, information was obtained from subjects on current health status, medical history, drug prescriptions and actual use, and smoking behaviour by a trained interviewer using a computerized questionnaire. Subjects were divided into three groups of smoking behaviour: current smokers, former smokers and those who had never smoked. A history of cerebrovascular event was considered positive when the diagnosis had been made by a physician and the diagnosis of myocardial infarction was based on ECG.

At the research centre several measurements were performed as part of the baseline data collection. The average of two blood pressure measurements, separated by a count of the pulse rate, taken in the sitting position at the right upper arm with a randomzero sphygmomanometer was used for the analysis [19]. Hypertension was defined as systolic blood pressure of $\geq 160 \mathrm{mmHg}$ and/or diastolic blood pressure $\geq 95 \mathrm{mmHg}$, and/or by the use of antihypertensive drugs for the indication hypertension. The collection of blood samples and subsequent procedures, and determination of serum total cholesterol, high-density lipoprotein cholesterol, plasma fibrinogen levels, factor VIIc and factor VIIIc activity, have been described in detail previously [9].

Ultrasonography of both carotid arteries was performed with a 7.5-MHz linear array transducer using a Duplex scanner (ATL Ultramark IV, Advanced Technology Laboratories, Bethel, Washington, USA), according to the Rotterdam Study scanning protocol [20]. The distance between the lumen-intima interface and the media-adventitia interface of the far wall represents the intimamedia thickness [21]. The interfaces of the distal common carotid artery were marked with a cursor over a length of $10 \mathrm{~mm}$. The beginning of the dilatation of the distal common carotid artery served as a reference point for the start of the measurement. The average of the intima-media wall thickness of each of three frozen images stored on video tape was taken as a measure for the current wall thickness of the distal common carotid artery. In addition, for each subject the total intima-media wall thickness was calculated as the average of the left and right side. Results from a reproducibility study on intima-media thickness measurements have been described elsewhere [22]. The carotid artery was also evaluated for the presence of atherosclerotic plaques, defined as focal widening relative to adjacent segments, with protrusion into the lumen composed either of only calcified deposits or of a combination of calcification and non-calcified material. A reproducibility study for the assessment of plaques in the carotid bifurcation showed moderate agreement [23].

SPET. Regional CBF studies were performed with $99 \mathrm{mTc}-$ HMPAO, prepared by adding $1110 \mathrm{MBq}$ freshly eluted sodium pertechnetate to a vial containing freeze-dried $0.5 \mathrm{mg} d, l$ HMPAO, $7.6 \mu \mathrm{g}$ stannous chloride dihydrate and $4.5 \mathrm{mg}$ sodium chloride (Ceretec). From this mixture, $740 \mathrm{MBq}$ was intravenously administered within 15 min after preparation. SPET scanning was carried out 10-20 min later. Data acquisition was performed with either a single-headed rotating gamma camera (Orbiter by Siemens) with a low-energy all-purpose collimator $(n=36)$, or a three-headed rotating gamma camera (Prism 3000 by Picker; $n=24$ ). Total acquisition angle was $360^{\circ}, 60$ projections of $30 \mathrm{~s}$ each, with a $64 \times 64$ matrix and spatial resolution for tomographic images of approximately $12 \mathrm{~mm}$ for the Siemens camera (estimated by the calculation of the full-width at half-maximum for $99 \mathrm{mTc}$ using an air distance between the object and rotating collimator of $15 \mathrm{~cm}$ ), and a $128 \times 128$ matrix and approximately $7 \mathrm{~mm}$ spatial resolution for the Picker camera, as estimated by the manufacturer. We reconstructed transversal (parallel to orbitomeatal line) slices, after correction for attenuation, by calculation of the geometric means. The geometric mean was calculated giving weight to each variable relative to the distance to the outer cortex.

Regions of interest. Regions of interest (ROIs) were drawn by a standardized procedure on a personal computer with GAMMAPC 1.41 software (Dr. O. Nickel, Department of Nuclear Medicine, University of Mainz, Germany), as described previously [24, 25] The matrix of images acquired with the Picker camera was reduced from $128 \times 128$ to $64 \times 64$. The reference slice included one cerebellar hemisphere that contained a pixel with the highest counts of the entire cerebellum. After drawing a region by hand roughly around this hemisphere, the computer precisely defined a subregion within this region, with activity higher than $70 \%$ of the maximum value in this slice. The boundary of this reference region corresponded to the anatomical boundary of the cerebellum with reference to an anatomical atlas [26]. The mean activity of this ROI in counts per pixel was regarded as the reference value and all other slices in a linear colour scale were set relative to this value. The ROIs corresponding to frontal, temporal, temporo-parietal and parietal cortices were drawn by hand, in all slices, with reference to an anatomical atlas [26]. The temporo-parietal region was defined as the transition area of the temporal and parietal cortex where no reliable differentiation between these two areas could be made. The outer border of these ROIs was placed at the first line of pixels with a fast decline in activity, assuming that this reflects the transition from brain tissue to cerebrospinal fluid. The inner border was set to a fixed distance of $2 \mathrm{~cm}$ from the outer border to approximate the area of grey matter in the brain. With this method our intra-observer variability in terms of mean counts per pixel in a specific ROI was $0.8 \%$. With the exception of the outermost slices, all slices were included in the analysis. Normalized rCBF was defined as the average counts per pixel within an ROI divided by that of the cerebellar reference slice and multiplied by 100 (percentage). The average of right and left ROIs was used for all cortical regions, since no differences between right and left ROIs were found.

Magnetic resonance imaging. MRI scans of the brain were performed with a 1.5-T Philips Gyroscan. T2-weighted axial images 
were obtained using multiple-slice spin-echo sequences with a repetition time of $2000 \mathrm{~ms}$ and an echo time of 50 and $100 \mathrm{~ms}$. The total volume of the ventricles and corresponding total brain volumes were measured according to a previously described procedure [10]. The perimeters of the entire brain and of the ventricles were identified on each axial slice containing the lateral ventricles, with the use of a light table and transparent paper. The areas were measured in pixel percentages by means of an IBAS 2000 (Zeiss-Kontron) and summed over all slices. The volume of the lateral ventricles was expressed as a percentage of the total brain volume (ventricle-to-brain ratio) $[27,28]$.

The presence and severity of white matter lesions were defined as described previously [10]. In short, a distinction was made between white matter lesions directly adjacent to the ventricles (periventricular lesions) and punctate or confluent lesions at some distance from the ventricles (focal lesions). Punctate lesions were dichotomized at fewer than five and five or more lesions [8]. Small caps on the horns of the lateral ventricles and pencil-thin lining around the ventricles were considered normal. The overall severity of white matter lesions was graded as follows. Normal scans (grade 0, no/slight white matter lesions) showed no or slight periventricular hyperintensity (small caps or pencil-thin lining), fewer than five focal lesions and no confluent lesions. Abnormal scans with moderate periventricular hyperintensity (caps on both anterior and posterior horns of the lateral ventricles, corpus only partly involved, not irregularly extending into the deep white mat- ter), five or more focal lesions, or both, but no confluent lesions, were classified as grade 1 (moderate white matter lesions). Abnormal grade 2 scans (severe white matter lesions) showed severe periventricular hyperintensity (irregularly extending into the deep white matter or marked areas of hyperintensity completely surrounding the lateral ventricles) or confluent lesions. For comparison of persons with and without cerebral white matter lesions, persons with grade 1 and grade 2 scans were combined.

Statistical analysis. The relations between various individual variables (vascular risk factors, indicators of atherosclerosis and cerebral white matter lesions) and normalized $\mathrm{rCBF}$ were examined with multiple linear regression analysis, with adjustments for age, sex, type of SPET camera and ventricle-to-brain ratio [29]. When a significant relation was found, an additional analysis was performed with quartiles of the distribution, using indicator variables [29]. The relation between cerebral white matter lesions and rCBF was also analysed for trend, using the variable grade of severity as an independent and rCBF as a dependent variable. For analyses with factor VIIc activity, subjects taking anticoagulant medication at the time were excluded. Regression coefficients [with $95 \%$ confidence interval $(\mathrm{CI})]$ are reported as a measure of the strength of the association between putative determinants and rCBF.

Table 1. Subject characteristics ${ }^{a}$

\begin{tabular}{lccc}
\hline & $\begin{array}{l}65-75 \text { years } \\
(n=33)\end{array}$ & $\begin{array}{l}76-85 \text { years } \\
(n=27)\end{array}$ & $\begin{array}{c}\text { All subjects } \\
(n=60)\end{array}$ \\
\hline Sex (men/women) & $16 / 17$ & $12 / 15$ & $28 / 32$ \\
CAMCOG & $94.2 \pm 6.8$ & $90.3 \pm 8.7$ & $92.5 \pm 7.9$ \\
MMSEb $^{\text {b }}$ & $28.7 \pm 1.2$ & $27.5 \pm 2.0$ & $28.2 \pm 1.7$ \\
Ventricle-to-brain ratio & $7.0 \pm 1.9$ & $8.3 \pm 1.6$ & $7.6 \pm 1.9$ \\
Systolic blood pressure (mmHg) & $132.2 \pm 19.3$ & $142.3 \pm 18.1$ & $136.7 \pm 19.3$ \\
Diastolic blood pressure (mmHg) & $69.5 \pm 11.4$ & $69.1 \pm 10.2$ & $69.3 \pm 10.7$ \\
History of hypertension $(n)$ & 6 & 7 & 13 \\
History of myocardial infarction $(n)$ & 1 & 5 & 6 \\
Anticoagulant therapy $(n)$ & 2 & 3 & 5 \\
Haematocrit $(1 / 1)$ & $0.41 \pm 0.04$ & $0.40 \pm 0.04$ & $0.40 \pm 0.04$ \\
Fibrinogen $(g / 1)$ & $2.8 \pm 0.7$ & $3.2 \pm 0.6$ & $3.0 \pm 0.7$ \\
Factor VIIc (IU/l) & $1.0 \pm 0.3$ & $1.0 \pm 0.3$ & $1.0 \pm 0.3$ \\
Factor VIIIc (IU/l) & $1.9 \pm 0.7$ & $2.1 \pm 0.7$ & $2.0 \pm 0.7$ \\
Cholesterol (mmol/l) & $6.5 \pm 1.5$ & $6.5 \pm 1.2$ & $6.5 \pm 1.3$ \\
HDL (mmol/l) & $1.2 \pm 0.3$ & $1.2 \pm 0.4$ & $1.2 \pm 0.3$ \\
Intima-media vessel wall thickness $(\mathrm{mm})$ & $80.2 \pm 18.2$ & $92.5 \pm 25.9$ & $85.3 \pm 22.3$ \\
Plaques in either carotid bifurcation $(n)$ & 16 & 15 & 31 \\
Frontal rCBF $(\%)$ & $84.9 \pm 5.6$ & $83.5 \pm 5.2$ & $84.2 \pm 5.4$ \\
Parietal rCBF $(\%)$ & $83.4 \pm 5.1$ & $81.3 \pm 5.9$ & $82.4 \pm 5.4$ \\
Temporo-parietal rCBF $(\%)$ & $79.3 \pm 6.4$ & $77.2 \pm 6.0$ & $78.4 \pm 6.3$ \\
Temporal rCBF $(\%)$ & $84.1 \pm 4.8$ & $81.7 \pm 4.3$ & $83.0 \pm 4.7$ \\
No/slight WML $(n)$ & 28 & 18 & 46 \\
Moderate WML $(n)$ & 3 & 5 & 8 \\
Severe WML $(n)$ & 2 & 4 & 6 \\
\hline & & & \\
\hline
\end{tabular}

a Values are reported as mean $\pm \mathrm{SD}$

b Cognitive test from the Cambridge Examination for Mental disorders of the Elderly (CAMDEX) [49, 50] and Mini-Mental Status Examination (MMSE) [51]

${ }^{c}$ White matter lesions (WML). No/slight: $<5$ focal lesions, no/slight periventricular hyperintensities and no confluent lesions. Moderate: $<5$ focal lesions, moderate periventricular hyperintensities and no confluent lesions, or $\geq 5$ focal lesions, no/slight/moderate periventricular hyperintensities, and no confluent lesions. Severe: confluent lesions and/or severe periventricular hyperintensities 


\section{Results}

Characteristics of the study subjects are presented in Table 1 . Twenty-three percent of the subjects had white matter lesions while $10 \%$ had severe white matter lesions.

Analysis of blood pressure, independent of the effect of age, showed that both diastolic and systolic blood pressure were positively associated with normalized values of rCBF in all cortical regions, and these relations were statistically significant for temporo-parietal rCBF (Table 2). High fibrinogen levels were related to low normalized $\mathrm{rCBF}$ in all cortical regions, and a statistically significant relation was observed for parietal $\mathrm{rCBF}$ (Table 2). The relation of fibrinogen and parietal rCBF persisted after adjustment for previous myocardial infarction. Since current smoking is associated with increased levels of fibrinogen, these analyses were adjusted for current smoking. Smoking itself, defined in three groups of smoking behaviour, was not related to rCBF.
A threshold effect was observed for the relation of diastolic blood pressure and temporo-parietal rCBF in the additional analysis with quartiles of the distribution. Subjects in the lowest quartile of the distribution of diastolic blood pressure ( $\leq 60 \mathrm{mmHg}$ ) had a significantly lower temporo-parietal rCBF than subjects above the 25th percentile (difference $-5.1 \%$ rCBF; 95\% CI:-8.4\%,-1.8\%) (Fig. 1). Similar results were observed when subjects using antihypertensive drugs were excluded. Presence of hypertension was associated with higher normalized $\mathrm{rCBF}$ in all cortical regions, but none of these relations were statistically significant (Table 2). Subjects in the highest quartile of the distribution of fibrinogen $(>3.2 \mathrm{~g} / \mathrm{l}$ ) had significantly less parietal $\mathrm{rCBF}$ than subjects below the 75th percentile (difference $-4.4 \%$ rCBF; 95\% CI:-8.1,-0.7) (Fig. 2). No significant associations were found between factor VIIc, factor VIIIc and normalized rCBF (Table 2).

Measurements of the intima-media wall thickness of the carotid artery and plaques in either carotid bifurcation revealed lower normalized $\mathrm{rCBF}$ in all cortical re-

Table 2. Differences in rCBF in percentage relative to cerebellum according to levels of blood pressure, vascular risk factors, indicators of atherosclerosis and cerebral white matter lesions, adjusted for age, sex, ventricle-to-brain ratio and type of SPET camera ${ }^{a}$

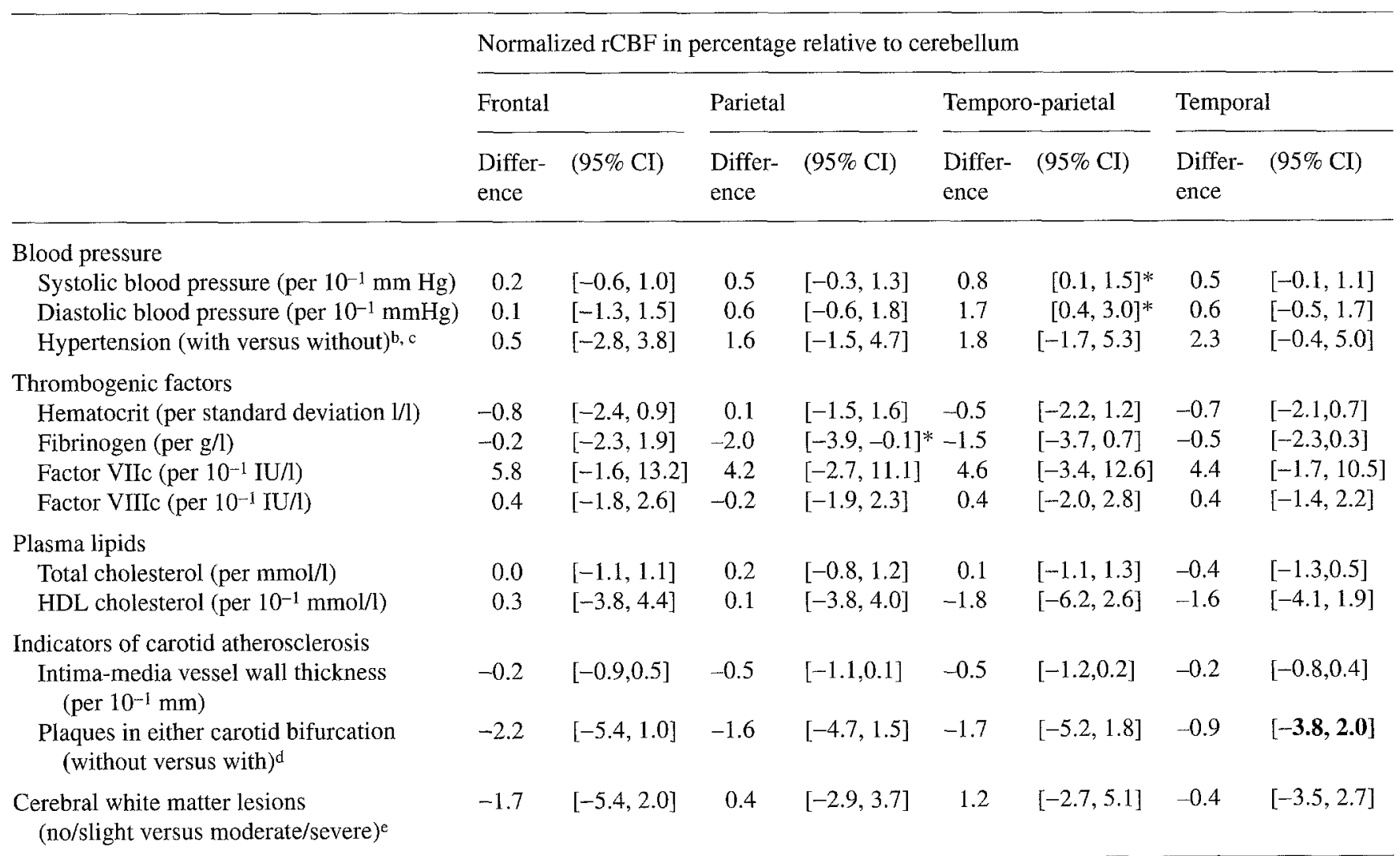

* Significant relation between independent variable and $\mathrm{rCBF}$ $(P<0.05)$

a Multiple linear regression coefficients are with $95 \%$ confidence intervals $(95 \% \mathrm{CI})$

b Defined as systolic blood pressure $\geq 160 \mathrm{mmHg}$ and/or diastolic blood pressure $\geq 95 \mathrm{mmHg}$, or use of antihypertensive medication c Differences in \% rCBF between persons with $(n=13)$ and without $(n=47)$ hypertension, respectively

d Differences in $\%$ rCBF between persons with $(n=31)$ and without $(n=16)$ plaques in either bifurcation of the carotid artery

e Differences in \% rCBF between persons with no/slight cerebral white matter lesions $(n=46)$ and persons with moderate/severe cerebral white matter lesions $(n=14)$ 
Quartiles diastolic blood pressure

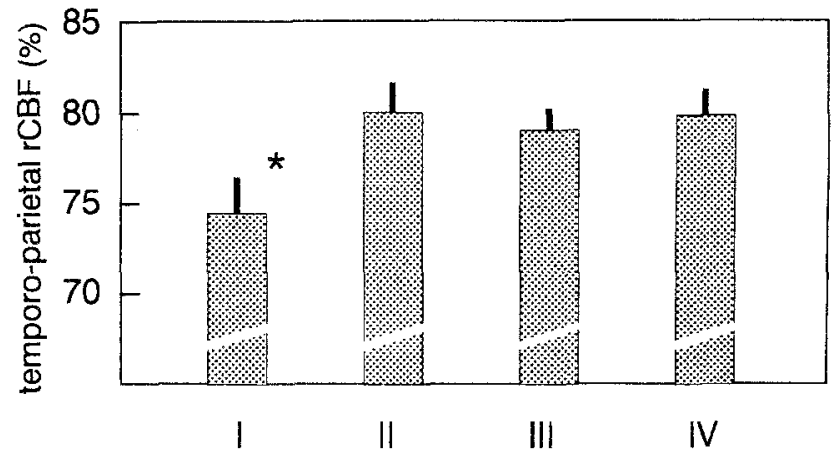

Fig. 1. Mean temporo-parietal rCBF values in percentage relative to cerebellum are shown for quartiles of diastolic blood pressure distribution. The 1st quartile is significantly different from the other quartiles with multiple linear regression, adjusted for age, sex, ventricle-to-brain ratio and type of SPET camera $(P<0.01)$

\section{Quartiles fibrinogen}

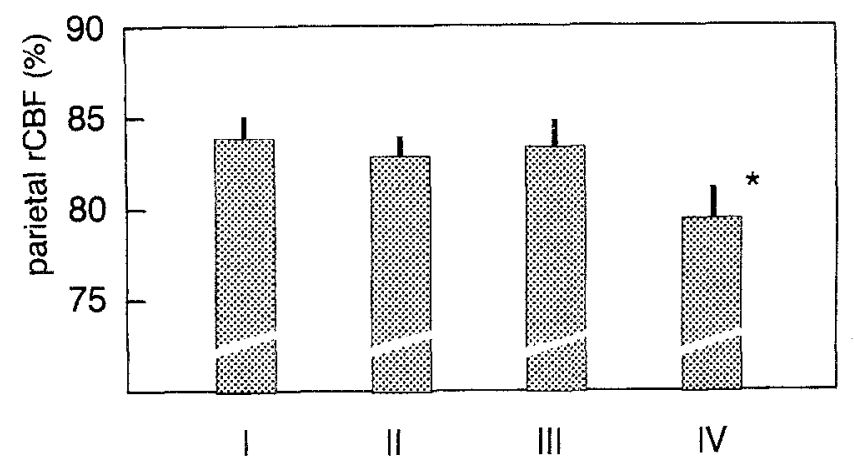

Fig. 2. Mean parietal rCBF values in percentage relative to cerebellum are shown for quartiles of fibrinogen distribution. The 4 th quartile is significantly different from the other quartiles with multiple linear regression, adjusted for age, sex, ventricle-to-brain ratio and type of SPET camera $(P<0.01)$

gions, but the inverse associations were not statistically significant (Table 2). Also when these relations were examined on either the right or the left side, similar results were obtained.

No consistent patterns of rCBF change with the presence of cerebral white matter lesions could be detected (Table 2). In persons with moderate or severe white matter lesions a decrease was observed in frontal $\mathrm{rCBF}$ while a slight increase was present in temporo-parietal rCBF. Using analyses for linear trend, these changes were not statistically significant.

\section{Discussion}

We studied the relation of vascular risk factors, indicators of atherosclerosis, cerebral white matter lesions and $\mathrm{rCBF}$ in a sample from the general population aged 65-85 years. The distribution of various demographic and vascular characteristics under study was very similar in the 60 subjects studied with SPET in this study to that in all subjects included in the original population in the same age range. Analysis of the relation between blood pressure and $\mathrm{rCBF}$ corrected by age revealed that both diastolic and systolic blood pressure were associated with temporo-parietal rCBF. A threshold effect of low temporo-parietal $\mathrm{rCBF}$ was observed with diastolic blood pressure under $60 \mathrm{mmHg}$ independent of the presence of hypertension. Increased atherosclerosis, indicated by measurements of the intima-media wall thickness of the carotid artery and plaques in the carotid bifurcation, was consistently associated with lower rCBF in all regions examined. However, these relations were not significant. Examination of thrombogenic factors in relation to $\mathrm{rCBF}$ showed that high levels of fibrinogen were independently associated with decreased $\mathrm{rCBF}$ in parietal cortex. In addition, no consistent relations between severity of cerebral white matter lesions and $\mathrm{rCBF}$ were observed.

The relation between blood pressure and temporo-parietal CBF in our study contrasts with results from previous studies, where no significant associations [30-33] or an inverse association [34] were found. Furthermore, hypertension has been associated with global CBF decrease [35], even in asymptomatic patients [2]. The discrepancies with results from our study are probably explained by differences in the study populations, since in previous studies few subjects over 65 years were included and persons were not selected from the general population. In addition, most of these studies used the ${ }^{133} \mathrm{Xe}$ inhalation method $[2,30-32,34,35]$ with a lower spatial resolution and reliability [36] than SPET with $99 \mathrm{mTc}$ HMPAO. Moreover, no statistical adjustment for age was made in a multiple comparison model in these studies. The role of atherosclerosis in $\mathrm{rCBF}$ changes in the healthy elderly is hard to compare with results from other studies since no direct quantified measures of atherosclerosis were taken in previous studies $[5,6]$. Indirect comparison is possible with those studies that have compared $\mathrm{rCBF}$ in persons with and without risk factors for vascular disease. The results of our study are in agreement with observations of reduced $\mathrm{rCBF}$ in those subjects with clinical evidence of atherosclerosis or increased vascular risk $[5,6]$ and with the finding that subjects with asymptomatic carotid artery disease have lower CBF values than asymptomatic subjects without abnormalities of the carotid artery [37].

The reduction in temporo-parietal $\mathrm{rCBF}$ in relation to blood pressure (the temporo-parietal region corresponding to the watershed area between the middle cerebral artery and the posterior cerebral artery) suggests that this region is most susceptible to changes in cerebral perfusion pressure, as previously reported [38]. In particular, our results are compatible with a shift of the lower limit of cerebral autoregulation to a higher pressure level in the elderly population [39-42]. Autoregulation is mainly a function of small arteries and arterioles $[39,43]$ and it has been suggested that atherosclerosis impairs the contractility of the muscle wall of these vessels [44]. It is 
tempting to speculate, based on this hypothesis of altered cerebral autoregulation, that one should be wary of low blood pressure levels in the elderly population. Further studies are needed, however, to determine precisely the clinical relevance of low temporo-parietal rCBF as related to low blood pressure levels.

The relation of cerebral white matter lesions and CBF in cerebral grey matter has been investigated in both symptomatic and asymptomatic study populations. In asymptomatic individuals, not selected by presence or absence of vascular risk factors, no significant CBF differences between subjects with and without white matter lesions were found [13-15]. In asymptomatic subjects, selected by the presence of cerebrovascular risk factors, two studies reported significantly decreased CBF in all cortical regions [11,32]. When symptomatic patients with atherosclerotic carotid artery disease were investigated, a global CBF reduction was observed in those with severe white matter lesions [12], and patients with vascular dementia of the Binswanger type showed decreased CBF compared to controls in frontal, temporal and parietal cortex [30]. With few reports available, these studies suggest that there is no, or only a weak inverse relation between cerebral white matter lesions and CBF in asymptomatic individuals that becomes stronger when this relation is examined in those with vascular risk factors and in symptomatic subjects. The absence of consistent rCBF changes in persons with moderate/severe cerebral white matter lesions in our study, where subjects were randomly sampled from the population and neurologically symptomatic individuals were excluded, fits well with these observations. Thus, together with data from previous studies, it appears that there are rCBF reductions related to cerebral white matter lesions in selected subjects with high risk of atherosclerotic vessel disease, as evidenced by the presence of vascular risk factors or by clinical symptoms, but not in those from the general population.

The inverse and independent relation of fibrinogen with parietal $\mathrm{rCBF}$ in our study is in agreement with the previously reported inverse relation of fibrinogen with global CBF in a heterogeneous sample of patients with vascular disease and controls [3] and in healthy persons over 45 years [45]. This is probably a result of lower blood flow velocity of the middle cerebral artery in subjects with high fibrinogen serum levels [46]. Further, the absence of some relations between indicators of vascular risk and $\mathrm{rCBF}$ fits well with previously reported results. Although Meyer et al. found a trend towards lower CBF values in normal subjects with elevated cholesterol and triglycerides, these relations were not significant [47]. A recent study showed that even long-lasting hypercholesterolaemia was not associated with alterations in $\mathrm{CBF}$ [48]. We found no significant alterations in rCBF as a function of haematocrit, which is consistent with a minimal relation of haematocrit with $\mathrm{CBF}$ in asymptomatic individuals as reported by Mathew et al. [34]. It appears that only in patients with cerebrovascu- lar disease is decreased CBF observed with higher levels of haematocrit, and these patients were excluded from our study [3].

In conclusion, both systolic and diastolic blood pressure are related to rCBF in temporo-parietal cortex, possibly due to altered cerebrovascular autoregulatory function. These findings may have clinical relevance for blood pressure control in the elderly population. In addition, high levels of fibrinogen are related to low rCBF in parietal cortex, possibly due to decreased blood flow velocity of the middle cerebral artery. Finally, cerebral white matter lesions in a sample from the general population appeared not to be related to $\mathrm{rCBF}$.

Acknowledgements. This work was supported in part by the NESTOR programme for geriatric research (Ministry of Health and Ministry of Education), the Netherlands Heart Foundation, the Netherlands Organization for scientific research (NWO) and the Municipality of Rotterdam. We are indebted to Dr E. Briët, Department of Haemostasis and Thrombosis Research, University Hospital Leiden, and to Dr H.H.D.M. van Vliet, Department of Haematology, University Hospital Rotterdam Dijkzigt, for the measurement of haemostatic variables; to T. Stehman and J. Vergeer for the other laboratory assessments; to all field workers for their skilful contributions to the data collection, in particular $\mathrm{H}$. Ensing and E. Herfst, who enthusiastically attended the study participants who went for SPET scanning; and to A. Reijs, J. van Peski, I. Loeve and M. Goemaat, Department of Nuclear Medicine, University Hospital Dijkzigt Rotterdam, for their assistance in data collection and data processing of SPET.

\section{References}

1. Naritomi H, Meyer JS, Sakai F, et al. Effects of advancing age on regional cerebral blood flow. Studies in normal subjects and subjects with risk factors for atherothrombotic stroke. Arch Neurol 1979; 36: 410-416.

2. Nobili F, Rodriguez G, Marenco $S$, et al. Regional cerebral blood flow in chronic hypertension: a correlative study. Stroke 1993; 24: 1148-1153.

3. Grotta J, Ackerman R, Correia J, et al. Whole blood viscosity parameters and cerebral blood flow. Stroke 1982; 13: 296-301.

4. Rogers RL, Meyer JS, Shaw TG, et al. Cigarette smoking decreases cerebral blood flow suggesting increased risk for stroke. JAMA 1983; 250: 2796-2800.

5. Dastur DK, Lane MH, Hansen D, B., et al. Effects of aging on cerebral circulation and metabolism in man. In: Birren JE, Butler RN, Greenhouse SW, Sokoloff L, Yarrow MR, eds. $\mathrm{Hu}$ man aging - a biological and behavioural study. Bethesda: U.S. Department of Health, Education, and Welfare, National Institute of Mental Health, DHEW publication no 986; 1963 : 57-76.

6. Shaw TG, Mortel KF, Meyer JS, et al. Cerebral blood flow changes in benign aging and cerebrovascular disease. Neurology $1984 ; 34: 855-862$.

7. Hunt AL, Orrison WW, Yeo RA, et al. Clinical significance of MRI white matter lesions in the elderly. Neurology 1989; 39: 1470-1474.

8. Mirsen TR, Lee DH, Wong CJ, et al. Clinical correlates of white-matter changes on magnetic resonance imaging scans of the brain. Arch Neurol 1991; 48: 1015-1021. 
9. Breteler MMB, van Swieten JC, Bots ML, et al. Cerebral white matter lesions, vascular risk factors, and cognitive function in a population-based study: The Rotterdam Study. Neurology 1994; 44: 1246-1254.

10. Breteler MMB, van Amerongen NM, van Swieten JC, et al. Cognitive correlates of ventricular enlargement and cerebral white matter lesions on magnetic resonance imaging. The Rotterdam Study. Stroke 1994; 25: 1109-1115.

11. Meguro K, Hatazawa J, Yamagushi T, et al. Cerebral circulation and oxygen metabolism associated with subclinical periventricular hyperintensity as shown by magnetic resonance imaging. Ann Neurol 1990; 28: 378-383.

12. Herholz K, Heindel W, Rackl A, et al. Regional cerebral blood flow in patients with leuko-araiosis and atherosclerotic carotid disease. Arch Neurol 1990; 47: 392-396.

13. Fazekas F, Niederkorn K, Schmidt R, et al. White matter signal abnormalities in normal individuals: correlation with carotid ultrasonography, cerebral blood flow measurements, and cerebrovascular risk factors. Stroke 1988; 19: 1285-1288.

14. Kobari M, Meyer JS, Ichijo M. Leuko-araiosis, cerebral atrophy, and cerebral perfusion in normal aging. Arch Neurol 1990; 47: 161-165.

15. Kobayashi S, Okada K, Yamashita K. Incidence of silent lacunar lesion in normal adults and its relation to cerebral blood flow and risk factors. Stroke 1991; 22: 1379-1383.

16. Hofman A, Grobbee DE, de Jong PT, et al. Determinants of disease and disability in the elderly: the Rotterdam Elderly Study. Eur J Epidemiol 1991; 7: 403-422.

17. Breteler MMB, van den Ouweland FA, Grobbee DE, et al. A community-based study of dementia: the Rotterdam Elderly Study. Neuroepidemiology 1992; 11 Suppl 1: 23-28.

18. McKhann G, Drachman D, Folstein M, et al. Clinical diagnosis of Alzheimer's disease: report of the NINCDS-ADRDA work group under the auspices of department of health and human services task force on Alzheimer's disease. Neurology 1984; 34: 939-944.

19. Joint National Committee on High Blood Pressure. 1988 report of the Joint National Committee on detection, evaluation, and treatment of high blood pressure. Arch Intern Med 1988; 148: 1023-1038.

20. Bots ML, Hofman A, Grobbee DE. Common carotid intimamedia thickness and lower extremity arterial atherosclerosis. The Rotterdam Study. Arterioscler Thromb 1994; 14: 1885-1891.

21. Pignoli P, Tremoli E, Poli A, et al. Intimal plus medial thickness of the arterial wall: a direct measurement with ultrasound imaging. Circulation 1986; 74: 1399-1406.

22. Bots ML, Mulder PGH, Hofman A, et al. Reproducibility of carotid vessel wall thickness measurements. The Rotterdam Study. J Clin Epidemiol 1994; 47: 921-930.

23. Bots ML, van Swieten JC, Breteler MMB, et al. Cerebral white matter lesions and atherosclerosis in the Rotterdam Study. Lancet 1993; 341: 1232-1237.

24. Claus JJ, van Harskamp F, Breteler MMB, et al. Assessment of cerebral perfusion with single-photon emission tomography in normal subjects and in patients with Alzheimer's disease: effects of region of interest selection. Eur J Nucl Med 1994; 21: 1044-1051.

25. Claus JJ, van Harskamp F, Breteler MMB, et al. The diagnostic value of SPECT with Tc-99m HMPAO in Alzheimer's disease: a population-based study. Neurology 1994; 44: 454. 461.

26. Aquilonius SM, Eckernas SA. A color atlas of the human brain. New York: Raven Press, 1980.
27. Gorelick PB, Chatterjee A, Patel D, et al. Cranial computed tomographic observations in multi-infarct dementia. A controlled study. Stroke 1992; 23: 804-811.

28. Kaye JA, DeCarli C, Luxenberg JS, et al. The significance of age-related enlargement of the cerebral ventricles in healthy men and women measured by quantitative computed X-ray tomography. J Am Geriatr Soc 1992; 40: 225-231.

29. BMDP statistical software manual. Berkeley: University of California Press, 1992.

30. Yao H, Sadoshima S, Kuwabara Y, et al. Cerebral blood flow and oxygen metabolism in patients with vascular dementia of the Binswanger type. Stroke 1990; 21: 1694-1699.

31. Brown MM, Wade JPH, Marshall J. Fundamental importance of arterial oxygen content in the regulation of cerebral blood flow in man. Brain 1985; 108: 81-93.

32. Isaka Y, Iiji O, Ashida $\mathrm{K}$, et al. Cerebral blood flow in asymptomatic individuals. Relationship with cerebrovascular risk factors and magnetic resonance imaging signal abnormalities. Jpn Circ J 1993; 57: 283-290.

33. Leenders KL, Perani D, Lammertsma AA, et al. Cerebral blood flow, blood volume and oxygen utilization: normal values and effect of age. Brain 1990; 113: 27-47.

34. Mathew RJ, Wilson WH, Tant SR. Determinants of regional cerebral blood flow in normal subjects. Biol Psychiatry 1986; 21: 907-914.

35. Meyer JS, Rogers RL, Mortel KF. Prospective analysis of long term control of mild hypertension on cerebral blood flow. Stroke 1985; 16: 985-990.

36. Anderson AR, Friberg HH, Schmidt JF, et al. Quantitative measurements of cerebral blood flow using SPECT and [99mTc]-d,l,-HM-PAO compared to xenon-133. J Cereb Blood Flow Metab 1988; 8: S69-S81.

37. Niederkorn K. Asymptomatic carotid artery disease detected by duplex scanning incidence and correlation with risk factors, cerebral blood flow and CT findings. Eur Neurol 1990; 30: 61-66.

38. Miller BL, Mena I, Daly J, et al. Temporo-parietal hypoperfusion with single-photon emission computed tomography in conditions other than Alzheimer's disease. Dementia 1990; 1 : $41-45$.

39. Strandgaard S, Paulson OB. Cerebral autoregulation. Stroke 1984; 15: 413-416.

40. Lartaud I, Makki T, Bray-des-Boses L, et al. Effect of chronic ANG I-converting enzyme inhibition on aging processes. IV. Cerebral blood flow regulation. Am J Physiol 1994; 267: R687-R694.

41. Lartaud I, Bray-des-Boscs L, Chillon JM, et al. In vivo cerebrovascular reactivity in Wistar and Fisher 344 rat strains during aging. Am J Physiol 1993; 264: H851-H858.

42. Shuaib A. Alteration of blood pressure regulation and cerebrovascular disorders in the elderly. Cerebrovasc Brain Metab Rev 1992; 4: 329-345.

43. Berne RM, Winn R, Rubio R. The local regulation of cerebral blood flow. Prog Cardiovasc Dis 1981; 24: 243-260.

44. Isaka Y, Okamoto M, Ashida K, et al. Decreased cerebrovascular dilatory capacity in subjects with asymptomatic periventricular hyperintensities. Stroke 1994; 25: 375-381.

45. Cavestri R, Radice L, Ferrarini F, et al. Influence of erythrocyte aggregability and plasma fibrinogen concentration on CBF with aging. Acta Neurol Scand 1992; 85: 292-298.

46. Ameriso SF, Paganini-Hill A, Meiselman HJ, et al. Correlates of middle cerebral artery blood velocity in the elderly. Stroke 1990; 21: 1579-1583. 
47. Meyer JS, Rogers RL, Mortel KF, et al. Hyperlipemia is a risk factor for decreased cerebral perfusion and stroke. Arch Neurol 1987; 44: 418-422.

48. Rodriguez G, Bertolini S, Nobili F, et al. Regional cerebral blood flow in familial hypercholesterolemia. Stroke 1994; 25 : 831-836.

49. Roth M, Huppert FA, Tym E, et al. CAMDEX, the Cambridge Examination for Mental Disorders of the Elderly. Cambridge, England: Cambridge University Press, 1988.
50. Derix MM, Hofstede AB, Teunisse S, et al. CAMDEX-N: the Dutch version of the Cambridge Examination for Mental Disorders of the Elderly with automatic data processing. Tijdschr Gerontol Geriatr 1991; 22: 143-150.

51. Folstein MF, Folstein SE, McHugh PR. 'Mini-mental state': a practical method for grading the cognitive state of patients for the clinician. I Psychiatr Res 1975; 12: 189-198. 